\title{
The Effect of the 2018 Economic Crisis on Purchasing Behavior
}

\author{
Eriş Ceylan and Ayça Can Kırgız
}

\begin{abstract}
Economic crisis is a reason for concern for every country in the world and it has a widespread impact on people. When we look at the economic crises in history, most of them were accompanied by high inflation, depreciation of money and rising unemployment rates. The negative impact of economic crisis on people's living standards also leads to changes in their consumption habits which is already a difficult task to predict. The aim of this study is to discuss the effects of the economic crisis that was started in 2018 and still ongoing, on purchasing behavior of the consumers in Turkey based on scientific data and methods. The results were interpreted by comparing them with the results of a previous study that has been conducted after 2008 economic crisis in Turkey. For this purpose, a survey which was prepared considering the possible effects of the crisis conducted on 456 consumers over the age of 18 and currently living in Turkey. The results were analyzed and discussed comparing groups of participants with different demographic information.
\end{abstract}

Index Terms-Consumption habits, economic crisis, purchasing behavior.

\section{INTRODUCTION}

Crises as a term is used in social sciences field in the meaning of "a sudden change for the worse", "tribulation", "depression" and "conjuncture" [1]. Crises arise from an interrelated series of events that happen in different time and places. These events act as a steppingstone to the next one, triggering each other and extending the scope of the resulting crisis [2].

Economic crises are events that closely concern all the countries of the world. Although in some cases they are considered as opportunities, they often have a wide range of negative effects on people. When we look at the economic crises in history, most of them were accompanied by high inflation, depreciation of money and rising unemployment rates. The negative impact on people's living standards can also lead to changes in their consumption habits.

It is vital for all companies to analyze consumer behavior and take appropriate actions. Nowadays, marketing departments as one of the most important departments of companies, look for ways to understand and respond to people's needs and desires. It is a complex and difficult process to predict consumer behavior even under normal circumstances. It becomes even more difficult in extraordinary periods such as economic crisis.

There have been more than 10 major economic crises in the history of Turkish Republic since the Great Depression

Published on June 9, 2020.

E. Ceylan, Istanbul Kent University, Turkey.

A. Kirgiz, Istanbul Kent University, Turkey.

(e-mail: ayca.cankirgiz@gmail.com) in 1929. Turkish economy has been dealing with many problems while making reforms in all areas. Dependence on foreign sources of financing and producing technology have usually been the main difficulty. When these difficulties became unmanageable, financial crises arose [6]. The crisis had devastating economic and social consequences in Turkey. Starting from the second half of 2008 through the last quarter of 2009, significant changes could be seen in the economic indicators. Exports started to fall, production and GDP also continually declined, and unemployment hit peak [4]. The crisis in 2008 affected the other industries and real economy very fast. In advanced capitalism, society is in a state of saturation in terms of consumption. It causes demand to extremely decrease when there is panic. It is a common situation in all advanced economies and indicates that real economy is vulnerable to economic crises. Even though the crisis originated from finance industry, whole system was already in chronic crisis [5].

After recent subprime mortgage crisis in 2008, US found the way out with monetary expansion. By the help of monetary expansion in global markets, Turkey enjoyed injecting the cheap foreign capital, to its growth model based on construction sector, that finally ran out of later in 2018. Turkish business did a lot of its borrowing in foreign currencies. However, Central Bank of the Republic of Turkey (TCMB) was pushed to hold lira interest rates on historic lows in a rich world after 2008. Although foreign currency loans were cheaper, they had potential risks for businesses that have lira income [3].

Turkey confronted a possible risk from developments in the US. The Federal Reserve (FED) started to raise interest rates from $0.2 \%$ in December 2015 to the point of $2.4 \%$ in December 2018. This rally encouraged investors to pull their capital out of emerging markets including Turkey. Turkey's foreign debt that deteriorate its economy and also increase vulnerabilities against foreign currency risks has two features: First, it's a high level of debt to refinance. Credit rating agency Fitch estimated that Turkey's total financing needs in 2018 was almost $\$ 230$ billion. Second, Turkish private sector had borrowed in foreign currency amounted to over $35 \%$ of GDP in 2018. Financing conditions become more expensive to repay with weaker lira [9]. As a result of monetary tightening in the US, as an importer country that gives trade deficit - Turkey's inflation figures reached to the level of $25 \%$ in 2018 . GDP growth declined by $3.0 \%$ in last quarter in 2018 and contracted by $2.6 \%$ in first quarter in 2019 when compared with last years' same periods. Therefore, Turkey's economy grew at $2.6 \%$ for the whole of 2018, from $7.4 \%$ in 2017 [8].

All economic crises negatively affect the shopping habits of consumers. The order of priority in consumption changes 
and is restricted. People's wishes are unlimited, their needs are limited. Especially in times of economic crisis, demands and needs have to be limited in changing.

\section{Methodology}

In this study, economic crisis phenomenon, the economic crisis started in Turkey in 2018 and the consumer decision process are explained. The aim of this study is to discuss the effects of the economic crisis that was started in 2018 and still ongoing, on purchasing behavior of the consumers in Turkey based on scientific data and methods. The results were interpreted by comparing them with the results of a previous study that has been conducted after 2008 economic crisis in Turkey.

The type of this research can be classified as descriptive qualitative. The method that was used in this study to obtain the necessary data is a survey consisting of 50 questions. The participants of the survey were defined as Turkish consumers that are older than 18 years old. Demographic questions such as gender, age, marital status, education level, profession, income, number of children and family size were included in the questionnaire form to make sure the sample properly represents the population. The questionnaire form was created online and conducted among 456 people. The questions of this survey were selected on the purpose of explaining the research topic properly. The types of the questions are 5-point Likert scale and ranking between different sectors reducing the spending. The data was analyzed using SPSS Statistics tool.

\section{A. Findings on Demographic Data}

The comprehensive demographic characteristics of this study are shown in table 1.

TABLE 1: DEMOGRAPHIC DATA

\begin{tabular}{|c|c|c|c|}
\hline Demographic variable & Value & Frequency & Percent \\
\hline \multirow{2}{*}{ Gender } & Male & 233 & 51,1 \\
\hline & Female & 223 & 48,9 \\
\hline \multirow{5}{*}{ Age } & $18-25$ & 55 & 12,1 \\
\hline & $26-33$ & 143 & 31,4 \\
\hline & $34-41$ & 74 & 16,2 \\
\hline & $42-49$ & 66 & 14,5 \\
\hline & Over 50 & 118 & 25,9 \\
\hline \multirow{2}{*}{ Marital Status } & Single & 177 & 38,8 \\
\hline & Married & 279 & 61,2 \\
\hline \multirow{6}{*}{ Education Level } & Primary ed. & 6 & 1,3 \\
\hline & High school & 31 & 6,8 \\
\hline & Assoc. degree & 15 & 3,3 \\
\hline & Bachelor's & 284 & 62,3 \\
\hline & Master's & 101 & 22,1 \\
\hline & Doctoral & 19 & 4,2 \\
\hline \multirow{8}{*}{ Occupation } & Student & 45 & 9,9 \\
\hline & Professional & 155 & 34 \\
\hline & Officer & 56 & 12,3 \\
\hline & Worker & & \\
\hline & $\begin{array}{l}\text { Mid-level } \\
\text { manager }\end{array}$ & 89 & 19,5 \\
\hline & $\begin{array}{l}\text { Senior } \\
\text { manager }\end{array}$ & 33 & 7,2 \\
\hline & Retired & 57 & 12,5 \\
\hline & Unemployed & 21 & 4,6 \\
\hline \multirow{2}{*}{ Household Income } & Under 5000 & 75 & 16,4 \\
\hline & $5001-10000$ & 146 & 32 \\
\hline
\end{tabular}

\begin{tabular}{|c|c|c|c|}
\hline & $10001-15000$ & 111 & 24,3 \\
\hline & $15001-20000$ & 58 & 12,7 \\
\hline & $20001-25000$ & 26 & 5,7 \\
\hline & Over 25000 & 40 & 8,8 \\
\hline \multirow{5}{*}{ Family Size } & 1 & 59 & 12,9 \\
\hline & 2 & 101 & 22,1 \\
\hline & 3 & 136 & 29,8 \\
\hline & 4 & 132 & 28,9 \\
\hline & $5+$ & 28 & 6,1 \\
\hline \multirow{5}{*}{ Number of Children } & None & 220 & 48,2 \\
\hline & 1 & 114 & 25 \\
\hline & 2 & 109 & 23,9 \\
\hline & 3 & 13 & 2,9 \\
\hline & $4+$ & 456 & 100 \\
\hline \multirow{5}{*}{ Number of Dependents } & 1 & 278 & 61 \\
\hline & 2 & 97 & 21,3 \\
\hline & 3 & 49 & 10,7 \\
\hline & 4 & 28 & 6,1 \\
\hline & $5+$ & 4 & 0,9 \\
\hline
\end{tabular}

\section{B. Findings on Reducing the Spending}

The results of the part that requires participants to rank sectors according to their spending reduction rates can be found in the Table 2 below. The participants, with a rate of $33,3 \%$ reduced their electronic expenses at the highest rate. Then, consumer durables, entertainment/vacation, clothing, personal care, education, and health sector follow. Food sector seems to be the sector where the participants reduced their expenditures at the least, with a rate of $45,4 \%$.

TABLE 2: FINDINGS ON SPENDING REDUCTION RATES

\begin{tabular}{|c|c|c|c|c|c|c|c|c|c|}
\hline & 1 & 2 & 3 & 4 & 5 & 6 & 7 & 8 & Total \\
\hline \multirow{2}{*}{ Food } & N 52 & 15 & 11 & 16 & 27 & 44 & 84 & 207 & 456 \\
\hline & $\% 11,4$ & 3,3 & 2,4 & 3,5 & 5,9 & 9,6 & 18,4 & 45,4 & 100,0 \\
\hline \multirow{2}{*}{ Personal care } & N 52 & 69 & 63 & 73 & 80 & 51 & 51 & 17 & 456 \\
\hline & $\% 11,4$ & 15,1 & 13,8 & 16,0 & 17,5 & 11,2 & 11,2 & 3,7 & 100,0 \\
\hline \multirow{2}{*}{ Electronic } & N 152 & 105 & 62 & 43 & 22 & 29 & 25 & 18 & 456 \\
\hline & $\% 33,3$ & 23,0 & 13,6 & 9,4 & 4,8 & 6,4 & 5,5 & 3,9 & 100,0 \\
\hline \multirow{2}{*}{$\begin{array}{l}\text { Consumer } \\
\text { durables }\end{array}$} & N 59 & 106 & 94 & 66 & 59 & 26 & 25 & 21 & 456 \\
\hline & $\% 12,9$ & 23,2 & 20,6 & 14,5 & 12,9 & 5,7 & 5,5 & 4,6 & 100,0 \\
\hline \multirow[t]{2}{*}{$\begin{array}{l}\text { Entertainment/ } \\
\text { Vacation }\end{array}$} & $\mathrm{N} 46$ & 55 & 78 & 90 & 77 & 63 & 28 & 19 & 456 \\
\hline & $\% 10,1$ & 12,1 & 17,1 & 19,7 & 16,9 & 13,8 & 6,1 & 4,2 & 100,0 \\
\hline \multirow[t]{2}{*}{ Clothing } & N 39 & 43 & 77 & 111 & 91 & 65 & 24 & 6 & 456 \\
\hline & $\% 8,6$ & 9,4 & 16,9 & 24,3 & 20,0 & 14,3 & 5,3 & 1,3 & 100,0 \\
\hline \multirow[t]{2}{*}{ Education } & N 31 & 30 & 46 & 34 & 63 & 125 & 90 & 37 & 456 \\
\hline & $\% 6,8$ & 6,6 & 10,1 & 7,5 & 13,8 & 27,4 & 19,7 & 8,1 & 100,0 \\
\hline \multirow[t]{2}{*}{ Health } & $\mathrm{N} 25$ & 33 & 25 & 23 & 37 & 53 & 129 & 131 & 456 \\
\hline & $\% 5,5$ & 7,2 & 5,5 & 5,0 & 8,1 & 11,6 & 28,3 & 28,7 & 100,0 \\
\hline
\end{tabular}

According to the rankings that were given by the participants, an average score point was also calculated for each sector. The ranking remains the same, except clothing and entertainment/vacation sectors get the same average scores.

\section{Factor Analysis}

Q.1.12 and Q1.13 were excluded from the analysis. Remaining 30 Likert type questions were analyzed, and 5 factors that explain $60,2 \%$ of the variables were determined as a result. The results of the factor analysis and the distribution of the questions to the factors can be seen in Table 3. 
TABLE 3: ROTATED COMPONENT MATRIX

\begin{tabular}{|c|c|c|c|c|c|c|c|c|}
\hline & \multicolumn{5}{|c|}{ FACTOR ANALYSIS } & \multirow[b]{2}{*}{$\begin{array}{l}\mathrm{Me} \\
\text { an }\end{array}$} & \multirow[b]{2}{*}{$\begin{array}{l}\text { Total } \\
\text { varian } \\
\text { ce } \\
\text { explai } \\
\text { ned }\end{array}$} & \multirow[b]{2}{*}{$\begin{array}{l}\text { Reliabi } \\
\text { lity } \\
\text { statistic } \\
\text { s }\end{array}$} \\
\hline $\begin{array}{l}\text { Cod } \\
e\end{array}$ & $\begin{array}{l}\text { Financ } \\
\text { ial } \\
\text { concer } \\
\text { ns }\end{array}$ & $\begin{array}{l}\text { Savin } \\
\text { gs } \\
\text { and } \\
\text { thrift }\end{array}$ & $\begin{array}{l}\text { Price } \\
\text { influe } \\
\text { nce }\end{array}$ & $\begin{array}{l}\text { Making } \\
\text { compari } \\
\text { son }\end{array}$ & $\begin{array}{l}\text { Negati } \\
\text { ve } \\
\text { thoug } \\
\text { hts }\end{array}$ & & & \\
\hline
\end{tabular}

$\begin{array}{ll}\text { Q1. } & \mathbf{0 , 7 6 8}\end{array}$

Q1. $\mathbf{0 , 7 3 3}$

$\begin{array}{ll}\text { Q1. } & \mathbf{0 , 7 1 7}\end{array}$

Q1. $\mathbf{0 , 6 8 5}$

\begin{tabular}{ll}
8 & $\mathbf{0 , 6 8 5}$ \\
\hline Q1. & $\mathbf{0 , 6 6 7}$
\end{tabular}

\begin{tabular}{ll}
10 & $\mathbf{0 , 6 6 7}$ \\
\hline Q1. & $\mathbf{0 , 6 0 1}$
\end{tabular}

Q3. $\mathbf{0 , 5 8 5}$

Q1. $\mathbf{0 , 5 4 4}$

Q1. $\quad 0,529$

\begin{tabular}{ll}
7 & $\mathbf{0 , 5 2 9}$ \\
\hline $\mathrm{Q} 1$. & $\mathbf{0 , 4 1 8}$
\end{tabular}

14

Q3. $\quad 0,744$

Q3.

\begin{tabular}{ll}
8 & $\mathbf{0 , 7 2 7}$ \\
\hline Q3. & $\mathbf{0 , 6 0}$
\end{tabular}

\begin{tabular}{ll}
15 & $\mathbf{0 , 6 0 4}$ \\
\hline Q3. & $\mathbf{0 , 5 0 7}$ \\
\hline
\end{tabular}

\begin{tabular}{ll}
16 & $\mathbf{0 , 5 0 7}$ \\
\hline $\mathrm{Q} 1$. & $\mathbf{0 , 4 9 2}$
\end{tabular}

$\begin{array}{ll}2 & \mathbf{0 , 4 9 2} \\ \text { Q3. } & \mathbf{0 , 4 5 5}\end{array}$

Q3. $\mathbf{0 , 4 3 4}$

$\begin{array}{lll}9 & \mathbf{0 , 4 3 4} & \\ \text { Q3. } & & \mathbf{0 , 7 6}\end{array}$

4

Q3

Q3.

Q3.

Q3.

Q3.

Q3.

Q3.

Q3.
6

Q3.

Q1

\begin{tabular}{|c|c|c|c|c|}
\hline $\begin{array}{l}\text { Q1. } \\
4\end{array}$ & $\mathbf{0 , 8 5 4}$ & \multirow{4}{*}{$\begin{array}{l}4,3 \\
9\end{array}$} & \multirow{4}{*}{8,971} & \multirow{3}{*}{0,765} \\
\hline $\begin{array}{l}\text { Q1. } \\
1\end{array}$ & $\mathbf{0 , 8 2 4}$ & & & \\
\hline $\begin{array}{l}\text { Q1. } \\
5\end{array}$ & 0,612 & & & \\
\hline $\begin{array}{l}\text { Q1. } \\
6\end{array}$ & 0,396 & & & \\
\hline
\end{tabular}

Kaiser-Meyer-Olkin Measure of Sampling Adequacy: 0,942

Bartlett's test: Approx. Chi-Square: 7584,495; Df: 435; Sig.: 0,000

To test the reliability of the factors, Cronbach Alpha test was applied. The overall alpha value for the data was calculated as 0,941 . Minimum required value for alpha changes from 0,6 to 0,7 depending on the source. To find out whether the data is suitable for factor analysis, KMO (Kaiser Mayer Olkin) and Bartlett's tests were applied. The $\mathrm{KMO}$ measure of sampling adequacy was calculated as 0,942 and the $p$ value was calculated as 0,000 . The results can be seen in Table 4 below.

TABLE 4: DeTAILED ANALYSIS OF THE FACTORS

\begin{tabular}{|c|c|c|c|}
\hline \multicolumn{4}{|c|}{ Detailed Analysis of The Factors } \\
\hline & & Mean & Std. \\
\hline \multirow{10}{*}{ 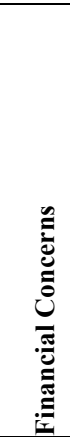 } & I use my belongings for a longer time. & 4,08 & 0,905 \\
\hline & $\begin{array}{l}\text { I think about financial matters more because of } \\
\text { the economic crisis. }\end{array}$ & 4,48 & 0,714 \\
\hline & $\begin{array}{l}\text { My purchasing behavior has changed due to the } \\
\text { economic crisis. }\end{array}$ & 4,25 & 0,837 \\
\hline & I am being more careful spending money. & 4,37 & 0,787 \\
\hline & I make more research before shopping. & 4,22 & 0,908 \\
\hline & I am being thriftier compared to the past. & 4,21 & 0,834 \\
\hline & $\begin{array}{l}\text { I postpone or totally give up on purchasing some } \\
\text { of my needs. }\end{array}$ & 3,99 & 0,968 \\
\hline & I am more cautious with using credit cards. & 3,98 & 1,04 \\
\hline & I am more cautious with borrowing loans. & 4,24 & 0,974 \\
\hline & I am more cautious with financial matters. & 4,39 & 0,693 \\
\hline \multirow{7}{*}{ 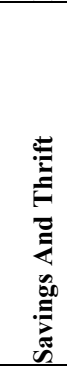 } & I spend more time at home than before. & 3,76 & 1,084 \\
\hline & $\begin{array}{l}\text { I compromise the brand while meeting my } \\
\text { needs. }\end{array}$ & 3,68 & 1,054 \\
\hline & $\begin{array}{l}\text { I compromise on quality while meeting my } \\
\text { needs. }\end{array}$ & 3 & 1,129 \\
\hline & $\begin{array}{l}\text { When shopping, I prefer products that I need } \\
\text { instead of flashy ones. }\end{array}$ & 4,26 & 0,769 \\
\hline & $\begin{array}{l}\text { Before buying a product, I consult with my } \\
\text { relatives more than before. }\end{array}$ & 3,44 & 1,08 \\
\hline & I go to shopping malls less than before. & 3,91 & 1,026 \\
\hline & I prefer farmers markets more than before. & 3,36 & 1,171 \\
\hline \multirow{5}{*}{ } & $\begin{array}{l}\text { Because of the high prices, I postpone } \\
\text { purchasing some products. }\end{array}$ & 4,43 & 0,714 \\
\hline & $\begin{array}{l}\text { Because of the high prices, I give up purchasing } \\
\text { some products. }\end{array}$ & 4,4 & 0,744 \\
\hline & $\begin{array}{l}\text { Price is a more decisive factor for me than } \\
\text { before. }\end{array}$ & 4,32 & 0,76 \\
\hline & I think longer before buying a product. & 4,24 & 0,74 \\
\hline & I now buy smaller quantities of products. & 3,9 & 0,928 \\
\hline \multirow{4}{*}{ 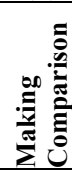 } & I follow special offers and discounts. & 4,07 & 0,948 \\
\hline & I'm spending more time on price research. & 4,16 & 0,844 \\
\hline & $\begin{array}{l}\text { I spend more time comparing products before } \\
\text { purchase. }\end{array}$ & 4,15 & 0,799 \\
\hline & I'm trying to get more out of promotions. & 4,12 & 0,853 \\
\hline \multirow{4}{*}{ 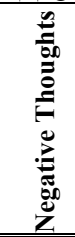 } & $\begin{array}{l}\text { Turkey is experiencing an economic crisis at the } \\
\text { moment. }\end{array}$ & 4,65 & 0,685 \\
\hline & $\begin{array}{l}\text { I think that the economic crisis will continue in } \\
2020 .\end{array}$ & 4,39 & 0,794 \\
\hline & I'm unhappy about the economic crisis. & 4,34 & 0,821 \\
\hline & $\begin{array}{l}\text { I think the economic crisis has affected my } \\
\text { business negatively. }\end{array}$ & 4,16 & 0,934 \\
\hline
\end{tabular}

D. Identifying And Testing The Hypotheses

To decide whether parametric or non-parametric tests should be applied to analyze the data, it is necessary to check whether the data is normally distributed or not.

TABLE 5: IDENTIFYING AND TESTING THE HYPOTHESES

\begin{tabular}{|c|c|c|c|c|c|c|c|}
\hline \multirow[b]{2}{*}{$\begin{array}{l}\text { Demogr } \\
\text { aphic } \\
\text { Veriable } \\
\text { s }\end{array}$} & \multirow[b]{2}{*}{$\begin{array}{l}\text { Te } \\
\text { st }\end{array}$} & \multicolumn{5}{|c|}{ Factors } & \multirow[b]{2}{*}{$\begin{array}{l}\text { Explic } \\
\text { ation }\end{array}$} \\
\hline & & $\begin{array}{l}\text { Finan } \\
\text { cial } \\
\text { concer } \\
\text { ns } \\
\end{array}$ & $\begin{array}{l}\text { Savin } \\
\text { gs and } \\
\text { thrift }\end{array}$ & $\begin{array}{l}\text { Price } \\
\text { influe } \\
\text { nce }\end{array}$ & $\begin{array}{l}\text { Makin } \\
\mathrm{g} \\
\text { compa } \\
\text { rison } \\
\end{array}$ & $\begin{array}{l}\text { Negati } \\
\text { ve } \\
\text { thoug } \\
\text { hts }\end{array}$ & \\
\hline \multirow[b]{2}{*}{ Gender } & $\begin{array}{l}\text { Sig. } \\
(p) \\
\end{array}$ & 0,222 & 0,940 & 0,733 & 0,240 & 0,242 & \multirow{2}{*}{$\begin{array}{l}\text { Hypoth } \\
\text { esis H0 } \\
\text { was } \\
\text { rejecte } \\
\text { d for } \\
\text { all } \\
\text { variabl }\end{array}$} \\
\hline & 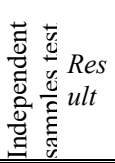 & $\begin{array}{l}\mathrm{p}>0,0 \\
5 \\
\text { No } \\
\text { Differ } \\
\text { ence } \\
\end{array}$ & $\begin{array}{l}\mathrm{p}>0,0 \\
5 \\
\text { No } \\
\text { Differ } \\
\text { ence } \\
\end{array}$ & $\begin{array}{l}\mathrm{p}>0,0 \\
5 \\
\text { No } \\
\text { Differ } \\
\text { ence } \\
\end{array}$ & $\begin{array}{l}\mathrm{p}>0,05 \\
\text { No } \\
\text { Differe } \\
\text { nce }\end{array}$ & $\begin{array}{l}\mathrm{p}>0,0 \\
5 \\
\text { No } \\
\text { Differ } \\
\text { ence } \\
\end{array}$ & \\
\hline
\end{tabular}




\begin{tabular}{|c|c|c|c|c|c|c|c|}
\hline & & & & & & & es. \\
\hline \multirow[b]{2}{*}{ Age } & \multirow{2}{*}{ 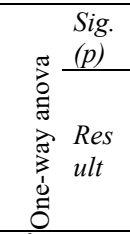 } & 0,000 & 0,297 & 0,00 & 0,181 & 0,089 & \\
\hline & & $\begin{array}{l}\mathrm{p}<0,0 \\
5 \\
\text { Differ } \\
\text { ence }\end{array}$ & $\begin{array}{l}\mathrm{p}>0,0 \\
5 \\
\text { No } \\
\text { Differ } \\
\text { ence }\end{array}$ & $\begin{array}{l}\mathrm{p}<0,0 \\
5 \\
\text { Differ } \\
\text { ence }\end{array}$ & $\begin{array}{l}\mathrm{p}>0,05 \\
\text { No } \\
\text { Differe } \\
\text { nce }\end{array}$ & $\begin{array}{l}\mathrm{p}>0,0 \\
5 \\
\text { No } \\
\text { Differ } \\
\text { ence }\end{array}$ & \\
\hline \multirow[b]{2}{*}{$\begin{array}{l}\text { Marital } \\
\text { Status }\end{array}$} & \multirow{2}{*}{ 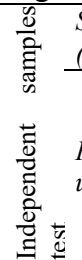 } & 0,073 & 0,214 & 0,618 & 0,842 & 0,129 & \multirow{2}{*}{$\begin{array}{l}\text { Hypoth } \\
\text { esis H0 } \\
\text { was } \\
\text { rejecte } \\
\text { d for } \\
\text { all } \\
\text { variabl } \\
\text { es. }\end{array}$} \\
\hline & & $\begin{array}{l}\mathrm{p}>0,0 \\
5 \\
\text { No } \\
\text { Differ } \\
\text { ence }\end{array}$ & $\begin{array}{l}\mathrm{p}>0,0 \\
5 \\
\text { No } \\
\text { Differ } \\
\text { ence }\end{array}$ & $\begin{array}{l}\mathrm{p}>0,0 \\
5 \\
\text { No } \\
\text { Differ } \\
\text { ence }\end{array}$ & $\begin{array}{l}\mathrm{p}>0,05 \\
\text { No } \\
\text { Differe } \\
\text { nce }\end{array}$ & $\begin{array}{l}\mathrm{p}>0,0 \\
5 \\
\text { No } \\
\text { Differ } \\
\text { ence }\end{array}$ & \\
\hline \multirow[b]{2}{*}{$\begin{array}{l}\text { Educatio } \\
\text { n Level }\end{array}$} & \multirow{2}{*}{ 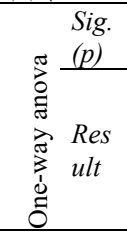 } & 0,298 & 0,000 & 0,122 & 0,04 & 0,324 & \\
\hline & & $\begin{array}{l}\mathrm{p}>0,0 \\
5 \\
\text { No } \\
\text { Differ } \\
\text { ence }\end{array}$ & $\begin{array}{l}\mathrm{p}<0,0 \\
5 \\
\text { Differ } \\
\text { ence }\end{array}$ & $\begin{array}{l}\mathrm{p}>0,0 \\
5 \\
\text { No } \\
\text { Differ } \\
\text { ence }\end{array}$ & $\begin{array}{l}\mathrm{p}<0,05 \\
\text { Differe } \\
\text { nce }\end{array}$ & $\begin{array}{l}\mathrm{p}>0,0 \\
5 \\
\text { No } \\
\text { Differ } \\
\text { ence }\end{array}$ & \\
\hline \multirow[b]{2}{*}{$\begin{array}{l}\text { Occupati } \\
\text { on }\end{array}$} & \multirow{2}{*}{ 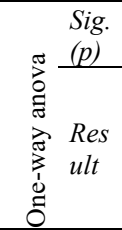 } & 0,038 & 0,005 & 0,057 & 0,034 & 0,424 & \\
\hline & & $\begin{array}{l}\mathrm{p}<0,0 \\
5 \\
\text { Differ } \\
\text { ence }\end{array}$ & $\begin{array}{l}\mathrm{p}<0,0 \\
5 \\
\text { Differ } \\
\text { ence }\end{array}$ & $\begin{array}{l}\mathrm{p}>0,0 \\
5 \\
\text { No } \\
\text { Differ } \\
\text { ence }\end{array}$ & $\begin{array}{l}\mathrm{p}<0,05 \\
\text { Differe } \\
\text { nce }\end{array}$ & $\begin{array}{l}\mathrm{p}>0,0 \\
5 \\
\text { No } \\
\text { Differ } \\
\text { ence }\end{array}$ & \\
\hline \multirow[b]{2}{*}{$\begin{array}{l}\text { Househo } \\
\text { ld } \\
\text { Income }\end{array}$} & \multirow{2}{*}{ 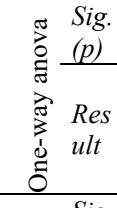 } & 0,013 & 0,000 & 0,016 & 0,228 & 0,004 & \\
\hline & & $\begin{array}{l}\mathrm{p}<0,0 \\
5 \\
\text { Differ } \\
\text { ence } \\
\end{array}$ & $\begin{array}{l}\mathrm{p}<0,0 \\
5 \\
\text { Differ } \\
\text { ence } \\
\end{array}$ & $\begin{array}{l}\mathrm{p}<0,0 \\
5 \\
\text { Differ } \\
\text { ence } \\
\end{array}$ & $\begin{array}{l}\mathrm{p}>0,05 \\
\text { No } \\
\text { Differe } \\
\text { nce }\end{array}$ & $\begin{array}{l}\mathrm{p}<0,0 \\
5 \\
\text { Differ } \\
\text { ence }\end{array}$ & \\
\hline \multirow[b]{2}{*}{$\begin{array}{l}\text { Family } \\
\text { Size }\end{array}$} & \multirow{2}{*}{ 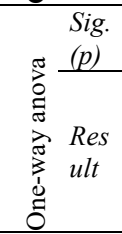 } & 0,326 & 0,306 & 0,806 & 0,459 & 0,009 & \\
\hline & & $\begin{array}{l}\mathrm{p}>0,0 \\
5 \\
\text { No } \\
\text { Differ } \\
\text { ence }\end{array}$ & $\begin{array}{l}\mathrm{p}>0,0 \\
5 \\
\text { No } \\
\text { Differ } \\
\text { ence }\end{array}$ & $\begin{array}{l}\mathrm{p}>0,0 \\
5 \\
\text { No } \\
\text { Differ } \\
\text { ence }\end{array}$ & $\begin{array}{l}\mathrm{p}>0,05 \\
\text { No } \\
\text { Differe } \\
\text { nce }\end{array}$ & $\begin{array}{l}\mathrm{p}<0,0 \\
5 \\
\text { Differ } \\
\text { ence }\end{array}$ & \\
\hline \multirow[b]{2}{*}{$\begin{array}{l}\text { Number } \\
\text { of } \\
\text { Children }\end{array}$} & \multirow{2}{*}{ 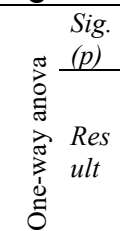 } & 0,000 & 0,003 & 0,000 & 0,654 & 0,059 & \\
\hline & & $\begin{array}{l}\mathrm{p}<0,0 \\
5 \\
\text { Differ } \\
\text { ence }\end{array}$ & $\begin{array}{l}\mathrm{p}<0,0 \\
5 \\
\text { Differ } \\
\text { ence }\end{array}$ & $\begin{array}{l}\mathrm{p}<0,0 \\
5 \\
\text { Differ } \\
\text { ence }\end{array}$ & $\begin{array}{l}\mathrm{p}>0,05 \\
\text { No } \\
\text { Differe } \\
\text { nce }\end{array}$ & $\begin{array}{l}\mathrm{p}>0,0 \\
5 \\
\text { No } \\
\text { Differ } \\
\text { ence }\end{array}$ & \\
\hline
\end{tabular}

\section{DISCUSSION AND CONCLUSION}

The participants of the survey were asked about their opinion on 32 statements. The statements were prepared with the purpose of comparing purchasing behavior with the pre-economic crisis period. As a result of the factor analysis that was applied to the statements in the questionnaire form, five factors were determined: financial concerns, savings and thrift, price influence, making comparison and negative thoughts.

Economic crisis causes people to worry more about financial issues than usual due to loss of value of current savings and the future savings being at risk. These financial concerns affect the consumers in a way that they are spending more carefully, being more cautious about borrowing and generally thinking more about financial matters. The participants agree on having more financial concerns than usual at a rate of 4,22 on 5,00 . As their age increases, their rate of having financial concerns increases as well. It can be related to having children or other dependents in older ages. The research shows that people with multiple children worry about financial matters more than people with one child, and the people with no children worry the least as it can be expected. In addition to that, as people get older, especially in the retirement period, their earnings may decrease considerably. This may cause them to feel more worried about financial issues.

In a country applying a current account deficit growth model, rising inflation due to increasing exchange rates makes people more sparing about money. People tend to be more careful about spending money to maintain their living standards in the face of personal financing conditions that has become more difficult. Minimum education group has the highest rate in this factor among the participants. They usually work in more basic jobs and their possibility of increasing their income in the future is lowest. It makes them more vulnerable to the changing economic conditions.

In a similar way, high prices caused by rising inflation force people to be more sensitive to price. With a rate of $4,26 / 5,00$ this factor is the second most important factor for the participants. According to research results, people postpone or totally give up on purchasing some of their needs. When the demographic characteristics of the participants were examined, there was no meaningful correlation with income level or number of children. Price increases affect people from all backgrounds in a different way regardless of the product. People have their own portfolio of products to purchase depending on their social status and income level and the prices of every portfolio has increased.

In times of economic crisis, people pay more attention to promotions and discounts. They start to spend more time comparing products and thinking before purchasing a product or service. Economic crisis means that economic conditions become more challenging. It represses the resources that people have. Therefore, people must spend their limited resources more effectively and efficiently. With a rate of 4,12/5,00 the participants agree on starting to do more research and thinking, independently from their backgrounds. $96 \%$ of the participants think that there is economic crisis in Turkey. $92 \%$ percent of the participants believe that it will continue in 2020. The economic crisis generally makes people unhappy and worried. Apart from the concerns about their finances and future, people fail to meet the requirements of having sustainable conditions to feel happy and socialized. To make things worse, the lack of confidence that political economic risks will improve in the short term has led to a decrease in people's confidence in economic management.

When the results were compared with the previous thesis, "An Appraisal on Customer Consuming Behavior During Economic Crisis", that was prepared in July 2009 after the global economic crisis, the following findings were obtained:

- In 2009, the sector where spending is reduced the most was vacation/entertainment. This year it became the third after electronics and consumer durables. The sectors where spending is reduced the less are food, health and education respectively in both researches.

- The score of financial concerns factor on a scale of 1 to 5 was 4,18 in 2009. It was found to be 4,22 in 2019, which means that people are equally concerned about 
financial matters.

- The score of savings and thrift factor on a scale of 1 to 5 was 3,95 in 2009. It was found to be 3,63 in 2019 , which means that people are less frugal in spending money.

- The score of price influence factor on a scale of 1 to 5 was 4,04 in 2009 . It was found to be 4,26 in 2019 , which means that people are more sensitive to price increases.

- The score of making comparison factor on a scale of 1 to 5 was 4,12 in 2009 . It was also found to be 4,12 in 2019, which means that people equally care about making product and price comparison.

- The score of negative thoughts factor on a scale of 1 to 5 was 3,80 in 2009 . It was found to be 4,39 in 2019 , which means that people have significantly more negative thoughts about the current economic crisis and its aftermath.

Since a great majority of the participants agree / strongly agree that the crisis will continue in 2020, a similar research can be done after a while to see the effects of a prolonged economic crisis on purchasing behavior. The findings of this study can be compared with the new results to further develop and improve it. Consumer behavior is a complex process which includes many different types of buying decisions that has many stages and that are influenced by a variety of social, cultural, personal, and psychological factors. People in different socioeconomic groups have different priorities and approaches towards the buying decisions during the economic crisis. However, the negative effects of the current economic crisis can be observed by looking at how the overall purchasing behavior of people is affected to a great extent.

\section{REFERENCES}

[1] C.C. Aktan, H. Şen, "Ekonomik kriz: nedenler ve çözüm önerileri," Yeni Türkiye Dergisi Ekonomik Kriz Özel Saylsl, vol. 2, no. 42, p. 1, 2000.

[2] G. Haşit, İşletmelerde kriz yönetimi ve türkiye'nin büyük sanayi işletmeleri üzerinde yapılan araştırma çalışması. Eskişehir: Anadolu Üniversitesi Yayınları, 2000.

[3] C, Koç, E. Ersoy. December 2018. How Turkey created a debt crisis. Bloomberg. [Online]. Available: https://www.bloomberg.com/news/features/2018-12-09/how-turkeycreated-a-debt-crisis.

[4] H. A. Kutlu, N. S. Demirci, "Küresel finansal krizi (2007-?) ortaya çıkaran nedenler, krizin etkileri, krizden kısmi çıkıs ve mevcut durum," Muhasebe ve Finansman Dergisi, vol. 1, no. 52, pp. 121-136, 2011.

[5] I. Önder, "Küresel kriz ve Türkiye ekonomisi," Muhasebe ve Finansman Dergisi, vol. 1, no. 42, pp.12-25, 2009.

[6] F. Özatay, "The 1994 currency crisis in Turkey," Journal of Policy Reform, vol. 3, no. 4, pp. 327-352, 2000.

[7] T. Pettinger. 2010. How the credit crisis spread [online]. Availale https://www.economicshelp.org/blog/1876/finance/how-the-crisisspread/

[8] L. Pitel. 2019. Turkey falls into recession as lira crisis takes longlasting toll. Financial Times. [Oonline] Available: https://www.ft.com/content/92818e7c-43c6-11e9- b168$96 \mathrm{a} 37 \mathrm{~d} 002 \mathrm{~cd} 3$

[9] A. Walker. August 2018. Is Turkey heading for an economic crisis. $B B C$ News. [Online]. Available: https://www.bbc.com/news/business45113472 .
A. KIRGIZ received her B.A. degree in Turkology with a focus on linguistics and Turkish / Cyrillic-based languages. She chose to pursue her M.A and Ph.D studies in the Department of Production Management and Marketing of the School of Economics and Administrative Sciences. Her linguistic background led her to focus on the Russian, China and Central Asia market. She has written several of articles and completed a lot of projects in the same field and has a book published in Palgrave Macmillan, one of the most prestigious publishing houses in England. It's name is "Green Marketing: A Case Study of the Sub-Industry in Turkey". In addition that, she has books published in Germany and Turkey and focused on branding in these books. Furthermore, she is the editor of series of marketing books. She is currently working as Associate Professor in the different universities and teaching Marketing, Brand Management, Sales Management and Integrated Marketing Communications. She has been working academically since 2005 . 